\title{
Compositional Imaging of Heterogeneous Materials with Single-Pass Kelvin Force Microscopy
}

\author{
S. N. Magonov
}

Agilent Technologies, 4330 W. Chandler Blvd, Chandler, AZ 85226, USA

The main functions of Atomic Force Microscopy (AFM) are high-resolution and low-force profiling of surface structures as well as the compositional imaging of heterogeneous materials. AFM can be applied to a broad range of materials in various environments (vacuum, air, vapors, liquids, etc) that uniquely differentiate this technique from other microscopic methods. The compositional mapping is based on the sensitivity of AFM-based techniques to mechanical, electric, thermal and other materials properties. As the stiffness of AFM probes is close to that of polymers, compositional imaging of heterogeneous polymer materials is often originated on differences of their mechanical properties. In such cases the individual constituents of the multicomponent polymers are best distinguished in phase images. The compositional imaging can be expanded using AFM-based electric measurements, and, specifically, Kelvin force microscopy (KFM). Simultaneous detection of the tip-sample mechanical and electrostatic interactions by amplitude modulation (AM) or frequency modulation (FM) modes at different frequencies is implemented in single-pass KFM. This technique, when applied in the intermittent contact operation with FM detection of electrostatic forces, enables the sensitive detection of surface potential with high spatial resolution [1]. The value of this approach was verified in studies of semiconductors, metals and self-assembled organic systems [2-3]. Identification of individual components in KFM is based on the surface potential dependence on work function, free electric charge, molecular dipole, and other electric properties. Furthermore, in the intermittent contact operation KFM is not affected by screening at high humidity, which expands the applicability of this method. The examples of the compositional imaging of different materials with KFM will be considered below.

KFM sensitivity to work function can be employed for studies of complex metal compounds, which typically do not provide strong mechanical contrast. The surface potential images of incomplete metal alloys $(\mathrm{Bi} / \mathrm{Sn})$ reveal the contrast coinciding with a difference of work functions of the metals. This is seen from the images in Fig. 1. In freshly prepared alloys samples the Bi-reach domains exhibit surface potential that is $0.2 \mathrm{~V}$ lower than $\mathrm{Sn}$ domains. This difference gradually diminishes when the sample is oxidized in air. Variations of the doping level are reflected in the surface potential images yet the quantitative absolute measurements are influenced by surface oxidation and probe contamination. The pronounced values of surface potential are observed on molecular systems with inherent dipole moment, especially, when the dipoles are oriented vertically [1]. Therefore, the fluorinated organic compounds including polymers might exhibit strong surface potential contrast thus aiding the identification of the fluorinated components in the complex materials.

Remarkably, the surface potential images of polystyrene and polymethylmethacrylate blends and block copolymers exhibit a contrast which reflects the distribution of the components. The contrast is noticeable only when the electrostatic force is stimulated with VAC $>3 \mathrm{~V}$. Most likely the electric field induces the dipole moments reflecting dielectric constants of the components. This effect explains the potential contrast between the components. The field-induced surface potential was also noticed at grain boundaries in single layers of thiophene and pentacene on $\mathrm{Si}$. This implies a field- 
induced polarization of a disordered material at grain boundaries. A proper choice of imaging environment can help compositional imaging. This has been proved in KFM studies of two important industrial polymers: the perfluorinated polymer (Nafion $\left.{ }^{\mathrm{TM}}\right)$, which is used in ion-exchange membranes, and the blend of intrinsically conducting polymer poly(3,4-ethylenedioxythiophene) with polystyrene sulfonate acid (PEDOT:PSS), Figs. 2-3. In both cases, the surface potential images, which were recorded in humid air, revealed 2-3 nm wide water channels in Nafion and hydrophilic PSS domains in the blend. Also in both cases the increased mobility of hydrophilic materials might play a role in the differentiation of the components in KFM.

\section{References}

[1] J. Alexander, S. Magonov, M. Moeller, J. Vac. Sci. Techn. B27 (2009) 903.

[2] S. Magonov, J. Alexander, S. Wu, “Advancing Characterization of Materials with Atomic Force Microscopy - Based Electric Techniques" in "Scanning Probe Microscopy of Functional Materials: Nanoscale Imaging and Spectroscopy" Eds. S. Kalinin, and A. Gruverman, Springer (2010) in press.

[3] S. Magonov, J. Alexander, S.-H. Jeong, N. Kotov, J. Nanosci. Nanotechn. (2010) in press.
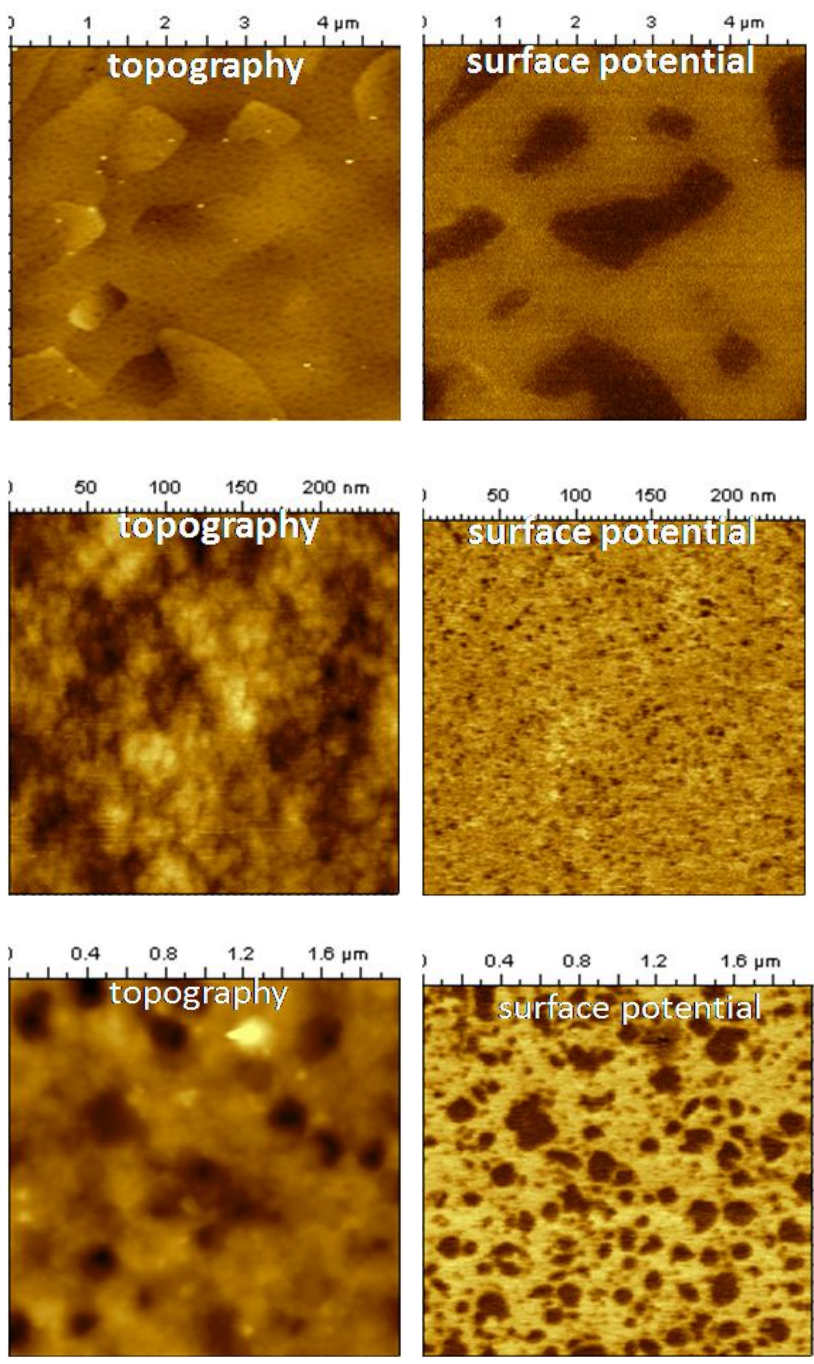

FIG 1. The KFM images of Bi/Sn alloy. The contrast covers the topography and surface potential variations in the $0-20 \mathrm{~nm}$ and $0-0.5 \mathrm{~V}$ ranges. The imaging was performed in air.

FIG 2. The KFM images of Nafion ${ }^{\mathrm{TM}}$ film. The contrast covers the topography and surface potential variations in the $0-5 \mathrm{~nm}$ and $0-0.4 \mathrm{~V}$ ranges. The imaging was performed at relative humidity above $95 \%$.

\section{FIG 3. The KFM images of PEDOT-PSS}

film. The contrast covers the topography and surface potential variations in the $0-100 \mathrm{~nm}$ and $0-0.4 \mathrm{~V}$ ranges. The imaging was performed at relative humidity above $95 \%$. 